\title{
Sapphire mirror for the KAGRA gravitational wave detector
}

\author{
Eiichi Hirose, ${ }^{1, *}$ Dan Bajuk, ${ }^{2}$ GariLynn Billingsley, ${ }^{3}$ Takaaki Kajita, ${ }^{1}$ Bob Kestner, ${ }^{2}$ Norikatsu Mio, ${ }^{4}$ \\ Masatake Ohashi, ${ }^{1}$ Bill Reichman, ${ }^{2}$ Hiroaki Yamamoto, ${ }^{3}$ and Liyuan Zhang ${ }^{3}$ \\ ${ }^{1}$ Institute for Cosmic Ray Research, the University of Tokyo, 5-1-5 Kashiwa-no-ha, \\ Kashiwa, Chiba 277-8582, Japan \\ ${ }^{2}$ Zygo Extreme Precision Optics, 3900 Lakeside Drive, Richmond, California 94806, USA \\ ${ }^{3}$ LIGO Laboratory, California Institute of Technology, MS 100-36, Pasadena, California 91125, USA \\ ${ }^{4}$ Photon Science Center, the University of Tokyo, 2-11-16 Bunkyo-ku, Hongo, Tokyo 113-8656, Japan
}

(Received 3 February 2014; published 24 March 2014)

\begin{abstract}
KAGRA, the Japanese interferometric gravitational wave detector currently under construction, will employ sapphire test masses for its cryogenic operation. Sapphire has an advantage in its higher thermal conductivity near the operating temperature $20 \mathrm{~K}$ compared to fused silica used in other gravitational wave detectors, but there are some uncertain properties for the application such as hardness, optical absorption, and birefringence. We introduce an optical design of the test masses and our recent R\&D results to address the above properties. Test polish of sapphire substrate has especially proven that specifications on the surface are sufficiently met. Recent measurements of absorption and inhomogeneity of the refractive index of the sapphire substrate indicate that the other properties are also acceptable to use sapphire crystal as test masses.
\end{abstract}

DOI: 10.1103/PhysRevD.89.062003

PACS numbers: 04.80.Nn, 95.55.Ym

\section{INTRODUCTION}

There are several ongoing interferometric gravitational wave projects in the world aiming for direct detection of gravitational waves [1-5]. Although each detector's design differs to some extent, they all use the same detection principle that distortion of spacetime will be coupled to phase change in the interferometer through test masses that also work as mirrors. When gravitational waves pass two free-falling test masses, their proper separation oscillates [6,7]. In reality, test masses are not free falling but are carefully suspended from a vibration isolation system so that the test masses can still follow geodesics. Since distortion of spacetime is so tiny, the detectors typically employ Fabry-Perot cavities to enhance the phase change. Fundamental noise sources that limit their sensitivity are seismic noise, thermal noise, and quantum noise. Among various techniques to reduce these noises, lowering the temperature of mirror-suspension system of the cavities is a way to reduce thermal noise, and KAGRA is the only detector that has taken this path so far (a proof-of-concept cryogenic detector CLIO [Cryogenic Laser Interferometer Observatory] is in Japan but is not currently operational [8]). Since it is necessary that test mass material has high thermal conductivity near the operating temperature $20 \mathrm{~K}$, KAGRA chose sapphire while the other detectors use fused silica. Sapphire, however, has a few disadvantages compared to fused silica in hardness, absorption, and birefringence. For our application, the effects of birefringence are minimized by choosing $\mathrm{C}$-plane as the mirror surface.

*hirose@icrr.u-tokyo.ac.jp
Absorption and inhomogeneity of refractive index arise from either impurities or defects in the crystal lattice, and these properties completely depend upon the quality of the crystal itself. Therefore, we investigated whether sapphire would be applicable to the gravitational wave detector. We address the KAGRA test mass design, results of a test polish, and properties of a sapphire bulk to discuss the feasibility of using sapphire crystal as test masses.

\section{FIELD PROPAGATION}

If mirrors in a Fabry-Perot cavity were perfectly fabricated, the only losses would be diffraction due to finite size of the mirror surface and transmitted light through end test masses (ETMs). In reality, there are losses originating from both substrate and coating. They are scattering due to surface aberration, which is commonly called either figure or roughness depending on its spatial frequency, scattering of coating, absorption of coating, scattering due to point defects, scratches, sleeks and so on. We do not count bulk absorption as a loss of a cavity. It is hard to predict scattering and absorption from coating layers theoretically, while the other loss sources from the substrate can be treated either numerically or analytically. We briefly explain how to estimate them to determine the specifications below. In the discussion, we assume a coated surface phase map. Propagation of the laser beams is approximated by the paraxial wave equation

$$
\frac{d^{2} E_{t}}{d x^{2}}+\frac{d^{2} E_{t}}{d y^{2}}+2 i k \frac{d E_{t}}{d z}=0
$$




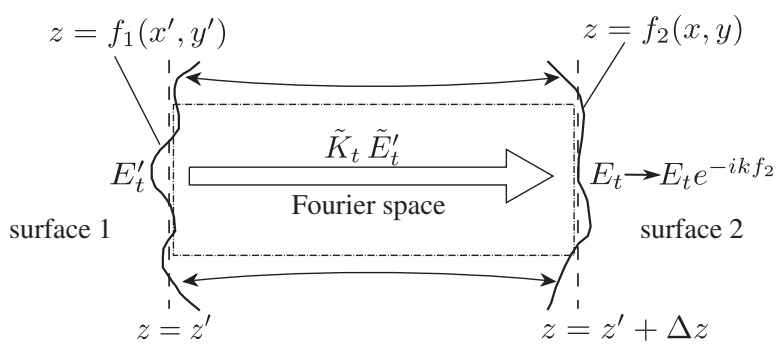

FIG. 1. Field propagation between two mirrors with aberration in a cavity. From $z=z^{\prime}$ to $z=z^{\prime}+\Delta z$, fast Fourier transform (FFT) technique is used, and phase change due to the arm length $\Delta z$, curvature, and surface aberration is taken care of later.

where $E_{t}$ is the slowly varying envelope of a field, $E(x, y, z)=\exp (-i k z) E_{t}(x, y, z)$, and $k$ is the wave number of the field. A general solution to the paraxial equation is the Huyghens integral, which is a convolution of the source field envelope $E_{t}^{\prime}=E_{t}\left(x^{\prime}, y^{\prime}, z^{\prime}\right)$ and the paraxial diffraction kernel $K(x, y, z)$ [9-11]:

$$
\begin{gathered}
E_{t}(x, y, z)=\iint d x^{\prime} d y^{\prime} E_{t}^{\prime} K(\Delta x, \Delta y, \Delta z) \\
K(x, y, z)=\frac{i}{\lambda z} e^{-i k \frac{x^{2}+y^{2}}{2 z}},
\end{gathered}
$$

where $\Delta x=x-x^{\prime}, \Delta y=y-y^{\prime}$, and $\Delta z=z-z^{\prime}$. In our case, $\Delta z$ is the arm length, and $\Delta x, \Delta y$ are the order of the mirror size at most. In Fourier space, this convolution becomes

$$
\tilde{E}_{t}(p, q, z)=\tilde{K}(p, q, \Delta z) \tilde{E}_{t}\left(p, q, z^{\prime}\right) .
$$

$E_{t}$ is given by the inverse Fourier transform of $\tilde{E}_{t}(p, q, z)$ :

$$
E_{t}(x, y, z)=\frac{1}{(2 \pi)^{2}} \iint d p d q e^{-i(p x+q y)} \tilde{E}_{t}(p, q, z) .
$$

FFT is a technique used to speed up the calculation of the field propagation [12-14]. For nonideal optics surfaces, we can treat them as deviations from the ideal surfaces. The field propagation inside the cavity is calculated in the following way. First, we apply FFT to convert $E_{t}^{\prime}$ to $\tilde{E}_{t}^{\prime}\left(p, q, z^{\prime}\right)$. Next, we multiply the diffraction kernel $\tilde{K}_{t}(p, q, \Delta z)$ with $\tilde{E}_{t}^{\prime}\left(p, q, z^{\prime}\right)$ in Fourier space to get the envelope distribution, $\tilde{E}_{t}(p, q, \Delta z)$. Then we inverse FFT $\tilde{E}_{t}(p, q, \Delta z)$ to have $E_{t}(x, y, \Delta z)$. Finally, we multiply phase change due to the surface profile of the mirror $e^{-i k f_{i}}$, where $f_{i}, i=1,2$ is a deviation from the ideal surface (see Fig. 1). There are several FFT based packages, and in this analysis a package named Stationary Interferometer Simulation (SIS) [13] was used. Spatial resolution of the FFT calculation is normally set as a few $\mathrm{mm}$ since the measured phase map of the entire mirror surface limits the resolution. For spatial frequencies below the spatial resolution, we rely on the golden rule, which relates the smooth-surface's total integrated scattering (TIS) to roughness in root-mean-square (rms) $\sigma[15,16]$ :

$$
\mathrm{TIS}=\left(\frac{4 \pi \sigma}{\lambda}\right)^{2}
$$

where $\lambda$ is the wavelength of the laser beam. In order to estimate loss due to point defects, scratches, and sleeks, we treat them as surface aberrations whose area and height are $S$ and $h$, respectively, and calculate a perturbed field under some assumptions $[17,18]$.

\section{DESIGN}

KAGRA is a dual-recycled Fabry-Perot Michelson interferometer [19], and the main parameters are summarized in Table I. The design of test masses is then to deliver the cavity by managing several loss sources inside. The size of the test mass is limited by an available high-quality $\mathrm{C}$-axis sapphire crystal in terms of purity, absorption, and homogeneity. The crystal is grown along the A-axis with a bigger size [20], but our choice to use C-plane as the mirror surface results in the size in Table I. Of course, a larger size is preferred in order to reduce thermal noise. The transmittance of the ETM will be counted as a loss. Table II shows the planned loss budget inside the cavity. For comparison, the advanced Laser Interferometer Gravitational Wave Observatory's (LIGO's) case is also listed $[18,21]$. Diffraction is a loss purely due to the finite size of the mirror, and for KAGRA it is two orders of magnitude smaller than $1 \mathrm{ppm}$. Scattering and absorption from coating layers really depend on quality of coating technology, so we set the numbers after discussion with a couple of coaters. We are planning to do coating tests with them to

TABLE I. Fabry-Perot cavity in KAGRA.

\begin{tabular}{lc}
\hline \hline Arm length & $3 \mathrm{~km}$ \\
Test mass diameter/thickness & $220 \mathrm{~mm} / 150 \mathrm{~mm}$ \\
Radius of curvature (ROC) & $1.9 \pm 0.0095 \mathrm{~km}$ \\
Loss in arm cavity & $100 \mathrm{ppm}$ a round trip \\
Transmission ETM/ITM & $7 \mathrm{ppm} / 0.004$ \\
Finesse & $\sim 1530$ \\
\hline \hline
\end{tabular}

TABLE II. Loss budget in the KAGRA arm cavity (ppm).

\begin{tabular}{lcc}
\hline \hline Loss sources & KAGRA & aLIGO \\
\hline Diffraction & $(1)$ & 1 \\
Defects & $1+1$ & $(0)$ \\
ETM transmission & 7 & 5 \\
Figure & $30+30$ & $20+20$ \\
Roughness & $5+5$ & $10+10$ \\
Point scattering & $9+9$ & $4+4$ \\
Absorption & $1+1$ & $0.5+0.5$ \\
\hline \hline
\end{tabular}


see if our loss budget is achievable. All that is left is how we break the rest down into defects, figure, and roughness. SIS, introduced in the previous section, is able to generate random surfaces with a rms roughness both inside and outside the clear aperture. We investigated a parameter space that includes the clear aperture, the rms error inside, and the rms error outside to see how much loss is generated in SIS with one-hundred pairs of randomly generated surfaces representing two mirrors. After trial and error, we decided to set the clear aperture as $140 \mathrm{~mm}$ in diameter. Figure 2 shows the distribution of loss in the arm cavity due to surface aberration, while Fig. 3 shows the accumulated fraction that gives us what fraction exists below a certain loss. We set the rms at $2 \mathrm{~nm}$ outside the clear aperture for all cases. These results convinced us to have a rms of $0.5 \mathrm{~nm}$ inside the clear aperture since it will be safe to say that loss due to figure error in the cavity is likely to be below $50 \mathrm{ppm}$. In this case, higher order mode fraction to the power inside the cavity is about $0.07 \mathrm{ppm}$. Adding $20 \%$ margin results in a $60 \mathrm{ppm}$ loss in the budget for figure error. This decision leaves $12 \mathrm{ppm}$ for micro roughness and

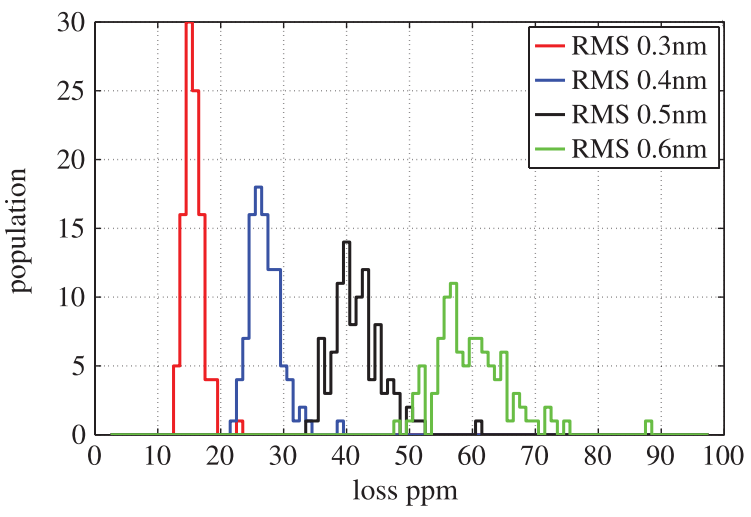

FIG 2 (color). Distribution of loss calculated by SIS due to randomly generated surfaces' aberration with a certain rms. In each rms, 100 pairs of surfaces were generated to make a Fabry-Perot cavity for the calculation.

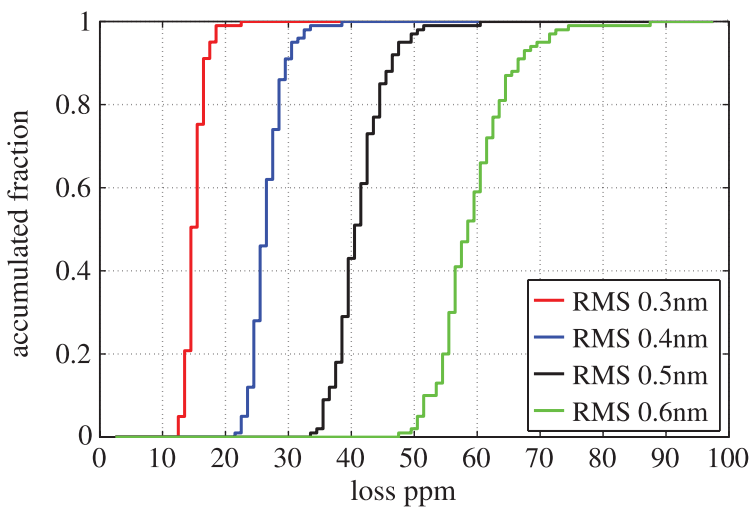

FIG 3 (color). Accumulated fraction of loss for each rms in Fig. 2. This shows what fraction of the population is below a certain loss. For rms $=0.5 \mathrm{~nm}, 98 \%$ of the calculated results are below $50 \mathrm{ppm}$. defects (in aLIGO, defects are included under roughness). We set the rms at $0.16 \mathrm{~nm}$ for high spatial frequency error above $1 \mathrm{~mm}^{-1}$, which should correspond to $3.6 \mathrm{ppm}$ according to Eq. (6). We count this loss as $5 \mathrm{ppm}$ per mirror to get about $40 \%$ margin (10 ppm total loss in the cavity). For simplicity, we count defects of radius greater than $2 \mu \mathrm{m}$ as point defects and defects of radius greater than $25 \mu \mathrm{m}$ as scratches. Defects of radius smaller than $2 \mu \mathrm{m}$ are controlled by the defect's density. For instance, a defect with radius $2 \mu \mathrm{m}$, depth $20 \mathrm{~nm}$, and density 0.25 gives a loss of $0.06 \mathrm{ppm}$. As for point like defects of radius $25 \mu \mathrm{m}$, the loss will be $0.02 \mathrm{ppm}$. We set the total number of those point defects less than 10 inside $100 \mathrm{~mm}$ diameter, and 50 over the entire surface, which corresponds to a loss of $0.4 \mathrm{ppm}$. For scratches, $0.5 \mathrm{ppm}$ corresponds to the total area $1.5 \times 10^{4} \mu \mathrm{m}^{2}$ that sets the upper limit over the entire surface area. Since the beam power is higher at central region, we set $2000 \mu \mathrm{m}^{2}$ scratches inside $100 \mathrm{~m}^{2}$ diameter, which corresponds to a loss of $0.064 \mathrm{ppm}$.

\section{TEST POLISH}

In the previous section, we described how to break the total cavity loss into several loss sources such as surface aberration. Irregularities of surface profile are directly related to specification of polishing, and we therefore investigated whether those specifications in figure error, roughness, and surface quality such as point defects, scratches, and sleeks could be achieved in reality. We performed a test-polish study with two sapphire substrates fabricated by GT Advanced Technologies as a pathfinder. One is their highest-grade crystal whose diameter is $100 \mathrm{~mm}$, while the other is a $200 \mathrm{~mm}$ diameter standard-grade one. The test polish was performed at Zygo Extreme Precision Optics (Zygo EPO) [22], and the results are summarized in Table III. The first six Zernike terms have been subtracted for figure rms and high spatial frequency (HSF) roughness rms. This operation is justified since these terms will disappear when mirrors are aligned to form a cavity. ROC and figure error were independently measured at both Zygo EPO and Caltech and most of the two measurements agree well, except the ROC. Figure 4 shows a phase map of the polished surface, measured at Zygo EPO. The surface was characterized to have the radius and figure error using a full aperture interferometer relative to a 12 inch transmission sphere whose radius was $2 \mathrm{~km}$. We chose $2 \mathrm{~km}$ intentionally instead of $1.9 \mathrm{~km}$ for convenience. The transmission sphere was calibrated to a high precision flat, and estimated accuracy for figure, radius, and astigmatism should be $0.15 \mathrm{~nm}, \pm 5 \mathrm{~m}$, and $0.2 \mathrm{~nm}$, respectively [23]. The subaperture interferometric measurement technique named phase measuring microscope (PMM) was used to measure high spatial frequency error of the surface using $2.5 \mathrm{x}$ and $50 \mathrm{x}$ microscope objectives. PMM is calibrated semiannually for each objective using a commercial target with National Institute of 
TABLE III. Results of test polish.

\begin{tabular}{lccc}
\hline \hline & ZYGO & CALTECH & Spec's \\
\hline @ $140 \mathrm{~mm}$ diameter & & & \\
Radius m & 2001.90 & 2004.61 & $2000 \pm 10$ \\
Figure rms nm & 0.24 & 0.21 & 0.5 \\
Astigmatism $Z_{2,2} n m$ & -0.41 & -0.13 & 3 \\
Astigmatism $Z_{2,-2} \mathrm{~nm}$ & 0.26 & 0.27 & 3 \\
@180 mm diameter & & & \\
Radius m & 2003.46 & 2006.34 & $\ldots$ \\
Figure rms nm & 0.48 & 0.45 & $\ldots$ \\
Astigmatism $Z_{2,2} n m$ & -0.68 & -0.30 & $\ldots$ \\
Astigmatism $Z_{2,-2} n m$ & 0.45 & 0.29 & $\ldots$ \\
HSF errors & & & \\
$1-750 \mathrm{~mm}{ }^{-1} \mathrm{~nm}$ & 0.11 & $\ldots$ & 0.16 \\
Surface quality & & & \\
(defects) & & & \\
Area @ $100 \mathrm{~mm} \mu \mathrm{m}^{2}$ & 0 & $\ldots$ & 2000 \\
Area @ $180 \mathrm{~mm} \mu \mathrm{m}^{2}$ & 14200 & $\ldots$ & 15000 \\
Point $>2 \mu \mathrm{m} @ 100 \mathrm{~mm}^{2}$ & 0 & $\ldots$ & 10 \\
Point $>2 \mu \mathrm{m} \mathrm{@} 200 \mathrm{~mm}^{-2}$ & 0 & $\ldots$ & 82 \\
Point density $<2 \mu \mathrm{mmm}^{-2}$ & 0 & $\ldots$ & 0.25 \\
\hline \hline
\end{tabular}

Standards and Technology traceable certifications. Figure 5 shows typical high spatial frequency error maps by PMM. The estimated accuracy of this high spatial frequency error should be less than rms $0.05 \mathrm{~nm}$. The surface was visually inspected for defects such as points, scratches, sleeks, and so on with both room light and a $150 \mathrm{~W}$ fiber light. Figure 6 shows the power spectral density (PSD) of the polished surfaces of both $100 \mathrm{~mm}$ and $200 \mathrm{~mm}$ substrates. The Zygo report for $200 \mathrm{~mm}$ substrate is a composite plot of three measurements, figure, PMM2.5x, and PMM50x, while the report for $100 \mathrm{~mm}$ substrate is one from two PMM measurements only. For low spatial frequency part (figure), two independent measurements done at Zygo and Caltech are in the plot and they agree fairly well. For comparison, fused silica data is on the plot, one of

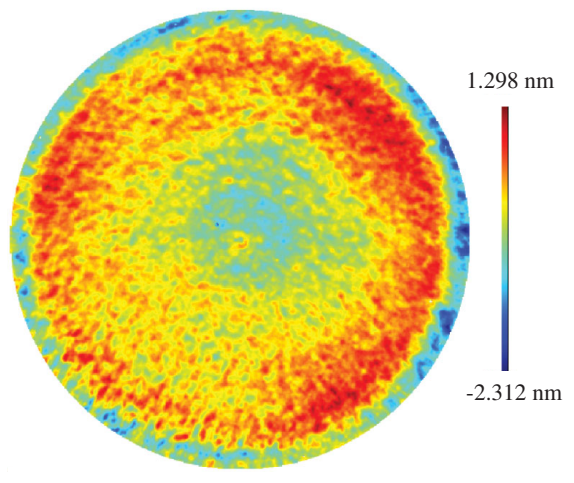

FIG. 4 (color). Phase map of figure error (low spatial frequency) measured at Zygo EPO (200 $\mathrm{mm}$ sapphire). The aperture size is $180 \mathrm{~mm}$ here. $\mathrm{rms}$ is $0.48 \mathrm{~nm}$ over the diameter and $0.24 \mathrm{~nm}$ over $140 \mathrm{~mm}$. Figure measurements done at Caltech using a different Fizeau interferometer showed almost the same results.
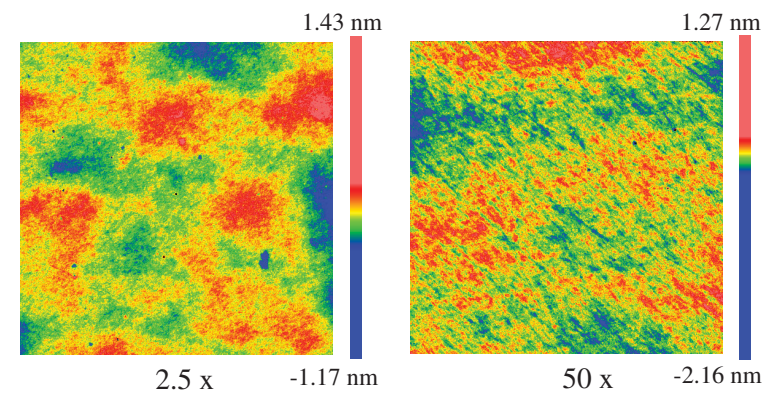

FIG. 5 (color). Phase maps of HSF error measured at Zygo EPO (100 mm sapphire) using PMM. The left is the result by $2.5 \mathrm{x}$ while the right is one by $50 \mathrm{x}$. The size of the map shown here is $4.9 \mathrm{~mm} \times$ $4.9 \mathrm{~mm}$ (left) and $0.5 \mathrm{~mm} \times 0.5 \mathrm{~mm}$ (right), respectively.

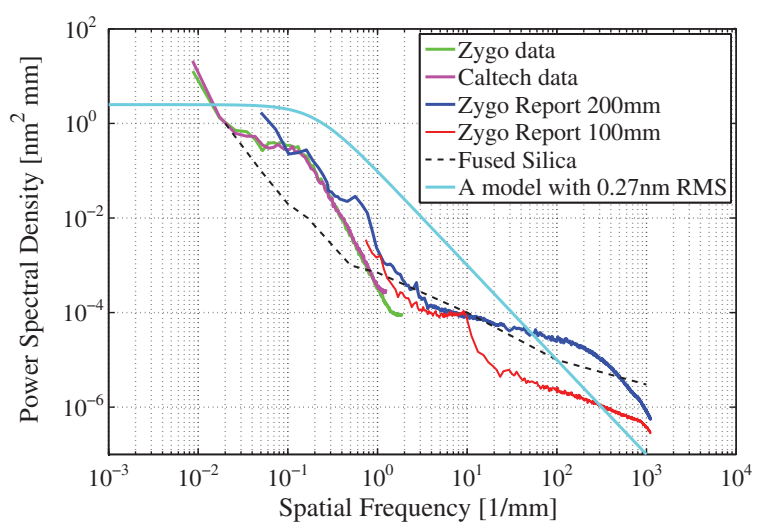

FIG. 6 (color). PSD plot of both $200 \mathrm{~mm}$ and $100 \mathrm{~mm}$ sapphire substrates. The Zygo report for $200 \mathrm{~mm}$ substrate is a composite plot of three measurements, figure, PMM2.5x, and PMM50x, while $100 \mathrm{~mm}$ substrate is one from only two PMM measurements only. For low spatial frequency part (figure), two independent measurements done at Zygo and Caltech agree fairly well. For comparison, fused silica data is in the plot, which is one of advanced LIGO's test mass data. We did not find disadvantages in terms of PSD. A model that gives $0.27 \mathrm{~nm}$ in rms when integrating from $0.01 \mathrm{~mm}^{-1}$ to $1 \mathrm{~mm}^{-1}$ is above our results.

advanced LIGO's test mass data. In this frequency band, fused silica is better there. We believe this is not a fundamental disadvantage of sapphire, but it just takes longer in polishing mainly because of the hardness of the material. For the high spatial frequency region, the high grade $100 \mathrm{~mm}$ sapphire's result is better than both the standard $200 \mathrm{~mm}$ sapphire and fused silica. Overall, we did not see disadvantages in terms of PSD in the spatial frequencies we explored. A model that gives a rms of about $0.27 \mathrm{~nm}$ when integrating from $0.01 \mathrm{~mm}^{-1}$ to $1 \mathrm{~mm}^{-1}$ is above our results, which makes sense given measured rms values.

\section{CRYSTAL PROPERTIES}

In this section, we discuss absorption and inhomogeneity of refractive index in the $100 \mathrm{~mm}$-diameter sapphire crystal 
used in the test-polish study. The absorption coefficient needs to be smaller than $50 \mathrm{ppm} / \mathrm{cm}$ in order for test masses to be cooled down to $20 \mathrm{~K}$ by the cryostat. Using photothermal common-path interferometer technique [24], we measured absorption of the bulk over the $50 \mathrm{~mm}$ central aperture. The interaction points were set $2 \mathrm{~mm}$ from a surface and data was taken every $1 \mathrm{~mm}$ step over the aperture to count the total number, 1961. Figure 7 shows a phase map taken in this method. The mean value is $43.4 \mathrm{ppm} / \mathrm{cm}$ and the standard deviation is $8.0 \mathrm{ppm} / \mathrm{cm}$ (see Fig. 8). Due to the nature of the relative measurement technique, there is some uncertainty about the absolute value, but the system's noise floor should be about rms $2 \mathrm{ppm} / \mathrm{cm}$. There is a clear line where absorption is higher than the other region. There is a clear correlation between absorption and distribution of refractive index in the bulk. The region of high absorption in Fig. 7 lines up where $d n$ is higher. We think of this as something related to lattice defect, and we are planning to work together with the crystal maker to tune some parameters in the fabrication process for lower absorption substrates. Inhomogeneity of refractive index is very important, especially for input test mass (ITM). If the index of refraction is not homogeneous inside the bulk, the ideal wavefront profile will be degraded to generate higher order modes when the beams propagate through it. There is a technique to compensate for this inhomogeneity of optical path difference, typically by polishing the back surface. We measured inhomogeneity of the refractive index of the sapphire bulk using the same full aperture interferometer at Caltech used to measure the figure of the $200 \mathrm{~mm}$-diameter sapphire. In order to kill the effect of surface profile of the substrate, we measured side 1 , side 2 , and side 2 through side 1 [25]. Figure 9 shows a phase map of inhomogeneity $d n \times t$ of the bulk over a $50 \mathrm{~mm}$ aperture, where $d n$ and $t$ are variations of the refractive index and thickness of the bulk, respectively. There is a similar line to one in Fig. 7 in the map where deviation is higher than the other region. After removing

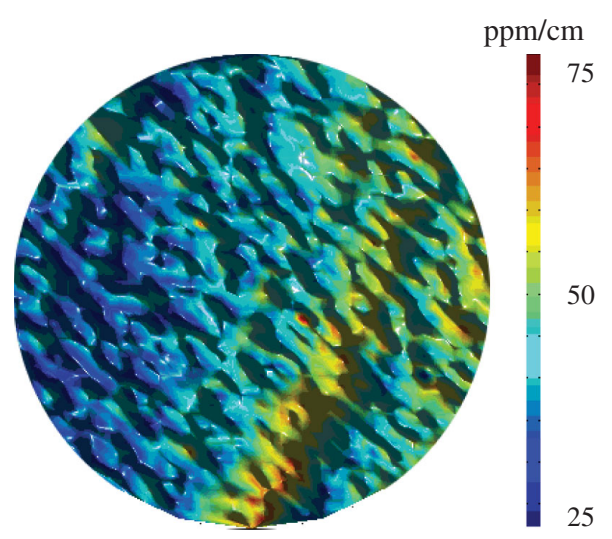

FIG. 7 (color). Absorption of $100 \mathrm{~mm}$ sapphire substrate in $\mathrm{ppm} / \mathrm{cm}$. The aperture size is $50 \mathrm{~mm}$ and the interaction point is $2 \mathrm{~mm}$ from the surface. There is a line where absorption is higher than the other regions.

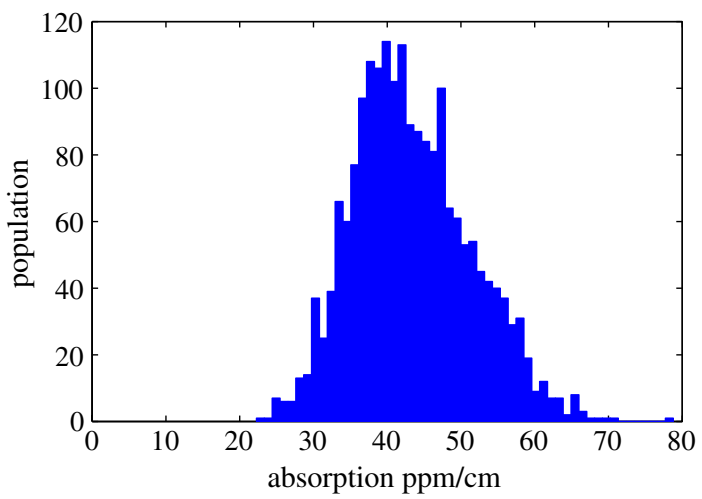

FIG. 8 (color online). Distribution of absorption in FIG. 7. The mean is $43.4 \mathrm{ppm} / \mathrm{cm}$ and the standard deviation is $8.0 \mathrm{ppm} / \mathrm{cm}$.

piston, tilt, and power terms, we had $P V 24.57 \mathrm{~nm}$ and rms $4.997 \mathrm{~nm}$. Given that the substrate is $60 \mathrm{~mm}$ thick, this corresponds to $d n=8 \times 10^{-8}$ over the aperture.

\section{DISCUSSION}

Although we are confident that the sapphire substrate can be polished to meet our surface specifications, we do still need to check whether coating specifications such as uniformity, scattering, and absorption are satisfied on sapphire substrates. Our current plan is to use multilayered dielectric coating of $\mathrm{SiO}_{2}$ and $\mathrm{Ta}_{2} \mathrm{O}_{5}$, and we have to make sure that optical properties are acceptable and the polished surface is actually preserved after coating in terms of radius of curvature, figure error, and so on. Otherwise, we would need to feed back the specifications of polishing to have smaller errors. The literature introduced a very low loss coating on a $30 \mathrm{~mm}$ diameter sapphire substrate [26], and the CLIO already demonstrated a loss less than $100 \mathrm{ppm}$ in the arm cavity whose test masses are $100 \mathrm{~mm}$-diameter sapphire mirrors [27]. We are planning to do a test-coating study using the substrates we described here to see if our low loss coating specifications are feasible. For the inhomogeneity of the refractive index, we think that we are at least able to reduce it by polishing the back surface to compensate up to the power term, as we processed data in the previous section. It is even possible to remove the structure due to the lattice distortion in Fig. 9 by a technique called Ion Beam Figuring. However, if the origin of the structure is deep inside the substrate, the phase map coming out of the back surface will be sensitive to alignment of the optic. And, considering the fact that test masses will move all the time, we would like to have the inhomogeneity as small as possible. Because of the correlation between absorption and inhomogeneity of the refractive index, we believe this direction should reduce the absorption level of the substrate. Therefore, it is essential to study both the structure of the refractive index and absorption inside the sapphire bulk to give us a hint for better crystal fabrication. According to discussion with the crystal maker, 


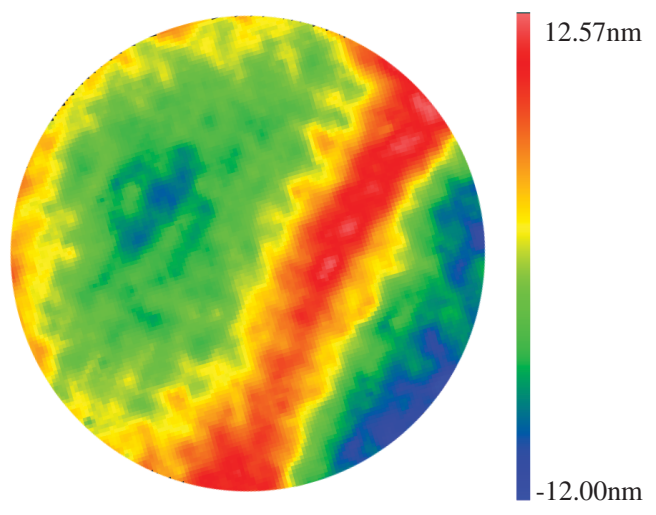

FIG. 9 (color). Phase map of homogeneity of refractive index. The unit is nm since data is product $d n \times$ thickness. We measured three wavefront data, side 1 , side 2 , and side 2 through side 1 . The last one contains information of refractive index of the bulk, and carefully subtracted information of both side 1 and side 2 . Then, terms up to power term were subtracted to bring the inhomogeneity to light. This is what will be left even if we have a spherical backsurface profile to compensate the inhomogeneity and the level is about $8 \times 10^{-8}$ after dividing by the thickness.

there are several parameters that can be tuned to control the growth of their sapphire crystal. If inhomogeneity of the refractive index were applied to one of the ITMs in the gravitational wave detector, the optical path difference generated through the ITM $\delta x$ would cause a fractional reduction of the detector's sensitivity by $(k \delta x)^{2} \sim 5 \times 10^{-3}$ [26]. We need a full interferometer simulation with real data for a more accurate discussion. Of course, the effect is not so small but it is fair to say that it is not disastrous. Another issue we should mention here is that all metrology is done in room-temperature conditions while test masses are cooled down to $20 \mathrm{~K}$ and suspended from a cryogenic suspension system. Therefore, we need to analyze effect of the environmental difference between lab and operating conditions. These issues are very important for the performance of the detector and are left for future work.

\section{CONCLUSION}

We addressed optical design of the cryogenic mirror for the KAGRA gravitational wave detector. We performed a test polish with $\mathrm{C}$-axis sapphire substrates and demonstrated that the specifications were satisfied. It turned out that the high-quality sapphire crystal would be usable as test masses even if absorption and inhomogeneity of the refractive index of real test mass crystal were equal to those of the bulk we tested. In order to have smaller absorption and a more homogeneous index of refraction, we need a better understanding of the sapphire crystal. Although we have some experience in coating on sapphire substrates, a study of test coating is planned to check whether our specifications are satisfied in reality.

\section{ACKNOWLEDGMENTS}

We are grateful for financial support from the Leadingedge Research Infrastructure Program funded by the Ministry of Education, Culture, Sports, Science and Technology (MEXT) in Japan. The LIGO Observatories were constructed by the California Institute of Technology and Massachusetts Institute of Technology with funding from the NSF under cooperative agreement No. PHY9210038. The LIGO Laboratory operates under cooperative agreement No. PHY-0328418. We thank our colleagues in both the KAGRA Collaboration and the LIGO Scientific Collaboration (LSC). This paper has document numbers JGW-P1302017 and LIGO-P1400005.
[1] LIGO Collaboration, http://www.ligo.caltech.edu/.

[2] VIRGO Collaboration, https://wwwcascina.virgo.infn.it/.

[3] GEO600, http://www.geo600.org/.

[4] KAGRA Collaboration, http://gwcenter.icrr.u-tokyo.ac.jp/.

[5] Einstein Telescope, http://www.et-gw.eu/.

[6] C. W. Misner, K. S. Thorne, and J. A. Wheeler, Gravitation (Freeman, San Francisco, 1973).

[7] P. R. Saulson, Fundamentals of Interferometric Gravitational Wave Detectors (World Scientific, Singapore, 1994).

[8] S. Miyoki, T. Uchiyama, K. Yamamoto, M. Ohashi, K. Kuroda, T. Akutsu, S. Kamagasako, N. Nakagawa, M. Tokunari, K. Kasahara, S. Telada, T. Tomaru, T. Suzuki, N. Sato, T. Shintomi, T. Haruyama, A. Yamamoto, D. Tatsumi, M. Ando, A. Araya, A. Takamori, S. Takemoto, H. Momose, H. Hayakawa, W. Morii, and
J. Akamatsu, Classical Quantum Gravity 23, S231 (2006).

[9] M. Born and E. Wolf, Principles of Optics (Cambridge University Press, Cambridge, England, 2002).

[10] A. E. Siegman, Lasers (University Science Books, Sausalito, CA, 1986).

[11] P. C. D. Hobbs, Building Electro-Optical Systems (Wiley, New York, 2009).

[12] J.-Y. Vinet, P. Hello, C. Man, and A. Brillet, J. Phys. I (France) 2, 1287 (1992).

[13] H. Yamamoto, Report No. LIGO-T070039-v7, 2011.

[14] T. Tomaru, S. Miyoki, M. Ohashi, K. Kuroda, T. Uchiyama, T. Suzuki, A. Yamamoto, T. Shintomi, A. Ueda, D. Tatsumi, S. Sato, K. Arai, K. W. Masaki Ando, K. Nakamura, M. Watanabe, K. Ito, I. Kataoka, H. Yamamoto, B. Bochner, and Y. Hefetz, Appl. Opt. 41, 5913 (2002). 
[15] J. Bennett and L. Mattsson, Introduction to Surface Roughness and Scattering (Optical Society of America, Washington, DC, 1999), 2nd ed.

[16] J. C. Stover, Optical Scattering: Measurement and Analysis (SPIE, Bellingham, Washington, USA, 2012), 3rd ed.

[17] H. Yamamoto, Report No. LIGO-T1000154-v5, 2010.

[18] H. Yamamoto, Report No. LIGO-G1300398-v2, 2013.

[19] Y. Aso, Y. Michimura, K. Somiya, M. Ando, O. Miyakawa, T. Sekiguchi, D. Tatsumi, and H. Yamamoto, Phys. Rev. D 88, 043007 (2013).

[20] C. P. Khattak, P. J. Guggenheim, and F. Schmid, Proc. SPIE 5078, Window and Dome Technologies VIII 5078, 47 (2003).

[21] H. Yamamoto, Report No. LIGO-P1200071, 2012.
[22] B. Reichman and R. Kestner, $200 \mathrm{~mm}$ Pathfinder Result Summary (Zygo ATP, Richmond, CA, USA, 2013).

[23] B. Reichman and R. Kestner, KAGRA $200 \mathrm{~mm}$ Pathfinder (Zygo ATP, Richmond, CA, USA, 2013).

[24] L. Zhang, G. Billingsley, and M. Phelps, Report No. LIGOE1000863-v2, 2011.

[25] B. F. Oreb, A. J. Leistner, G. Billingsley, W. P. Kells, and J. Camp, Proc. SPIE 4451, Optical Manufacturing and Testing IV 4451, 414 (2001).

[26] D. G. Blair, M. Notcutt, C. T. Taylor, E. K. Wong, C. Walsh, A. Leistner, J. Seckold, J.-M. Mackowski, P. Ganau, C. Michel, and L. Pinard, Appl. Opt. 36, 337 (1997).

[27] S. Miyoki (private communication). 